\title{
Linfoma canino: estudo caso-controle na Região Metropolitana do Recife-PE, Brasil
}

\author{
Canine lymphoma: a case control study in Metropolitan Region of Recife-PE, Brazil \\ Linfoma canino: estudio de caso controle en la Región Metropolitana de Recife-PE, Brasil
}

Recebido: 03/02/2022 | Revisado: 09/02/2022 | Aceito: 17/02/2022 | Publicado: 25/02/2022

Lidiana Carvalho de Holanda

ORCID: https://orcid.org/0000-0003-4744-6475

Universidade Federal Rural de Pernambuco, Brasil

E-mail: lidiana.holanda@icloud.com

Bruno Pajeú e Silva

ORCID: https://orcid.org/0000-0002-0040-393X Universidade Federal Rural de Pernambuco, Brasil E-mail: bruno_pajeu@ hotmail.com

José Wilton Pinheiro Júnior ORCID: https://orcid.org/0000-0002-0266-0956 Universidade Federal Rural de Pernambuco, Brasil E-mail: wilton.pinheiro@ufrpe.br

Andréa Alice da Fonseca Oliveira ORCID: https://orcid.org/0000-0002-3728-177X Universidade Federal Rural de Pernambuco, Brasil E-mail: andrea.oliveira@ufrpe.br

\begin{abstract}
Resumo
Objetivou-se avaliar os aspectos epidemiológicos e a distribuição espacial dos casos de linfoma canino na Região Metropolitana do Recife-PE, Brasil. Foram analisados os dados epidemiológicos obtidos de prontuários de 178 cães, sendo 89 casos e 89 controles. Para a análise dos fatores de risco foram consideradas informações relacionadas ao contato com carcinógenos, alimentação e moradia do animal e para confecção do mapa os pontos de georreferenciamento foram obtidos a partir dos municípios, bairros e códigos de endereçamento postal informados no prontuário clínico dos animais. Os fatores de risco identificados consistiram em contato com humanos em quimioterapia/radioterapia $(\mathrm{OR}=13,54 ; \mathrm{p}=0,014)$, alimentação mista $(\mathrm{OR}=5,59 ; \mathrm{p}=0,000)$, contato do animal com tintas e solventes $(\mathrm{OR}=5,08 ; \mathrm{p}=0,014)$, moradia próxima a avenida ou rua movimentada $(\mathrm{OR}=4,86 ; \mathrm{p}=0,000)$ e alimentação caseira $(\mathrm{OR}=2,44 ; \mathrm{p}=0,014)$. O maior número de casos de linfoma foi verificado na região sul do município de Recife, bem como na região norte de Olinda. A ocorrência de linfomas caninos diagnosticados na Região Metropolitana do Recife está intimamente relacionada à exposição de carcinógenos ambientais.
\end{abstract}

Palavras-chave: Cão; Carcinógenos; Epidemiologia; Georreferenciamento; Neoplasia linfóide.

\begin{abstract}
The objective of this study was to evaluate the epidemiological aspects and spatial distribution of cases of canine lymphoma in the Metropolitan Region of Recife-PE, Brazil. Epidemiological data obtained from medical records of 178 dogs were analyzed, including 89 cases and 89 controls. For the analysis of risk factors, information related to contact with carcinogens, food, and housing of the animal was considered. The map was prepared using georeferencing points obtained from municipalities, neighborhoods, and postal address codes in the clinical records of the animals. The risk factors identified consisted of contact with humans undergoing chemotherapy/radiotherapy $(\mathrm{OR}=13.54 ; \mathrm{p}=0.014)$, mixed feeding $(\mathrm{OR}=5.59 ; \mathrm{p}=0.000)$, animal contact with paints and solvents $(\mathrm{OR}=5.08$; $\mathrm{p}=0.014)$, living close to a busy avenue or street $(\mathrm{OR}=4.86 ; \mathrm{p}=0.000)$, and home-cooked meals $(\mathrm{OR}=2.44 ; \mathrm{p}=0.014)$. The highest number of lymphoma cases was found in the southern region of the city of Recife, as well as in the northern region of Olinda. The occurrence of canine lymphomas diagnosed in the Metropolitan Region of Recife is closely related to exposure to environmental carcinogens.
\end{abstract}

Keywords: Carcinogens; Dog; Epidemiology; Georeferencing; Lymphoid neoplasm.

\section{Resumen}

El objetivo de este estudio es evaluar los aspectos epidemiológicos y la distribución espacial de los casos de linfoma canino en la Región Metropolitana de Recife-PE, Brasil. Se analizaron los datos epidemiológicos obtenidos de las historias clínicas de 178 perros, 89 casos y 89 controles. Para el análisis de los factores de riesgo, se consideró la información relacionada con el contacto con carcinógenos, la alimentación y el alojamiento del animal y para realizar el mapa se obtuvieron los puntos de georreferenciación de los municipios, barrios y códigos postales informados en las historias clínicas de los animales. Los factores de riesgo identificados consistieron en el contacto con seres humanos sometidos a quimioterapia/radioterapia $(\mathrm{OR}=13,54 ; \mathrm{p}=0,014)$, la alimentación mixta $(\mathrm{OR}=5,59 ; \mathrm{p}=0,000)$, el 
contacto del animal con pinturas y disolventes ( $\mathrm{OR}=5,08 ; \mathrm{p}=0,014)$, el hecho de vivir cerca de una avenida o calle con mucho tráfico $(\mathrm{OR}=4,86 ; \mathrm{p}=0,000)$ y la alimentación en casa $(\mathrm{OR}=2,44 ; \mathrm{p}=0,014)$. El mayor número de casos de linfoma se encontró en la región sur de la ciudad de Recife, así como en la región norte de Olinda. La aparición de linfomas caninos diagnosticados en la Región Metropolitana de Recife está estrechamente relacionada con la exposición a carcinógenos ambientales.

Palabras clave: Perro; Carcinógenos; Epidemiología; Georreferenciación; Neoplasia linfoide.

\section{Introdução}

Os linfomas consistem em uma das neoplasias malignas mais frequentemente diagnosticadas nos cães e tratadas pela oncologia veterinária (Zandvliet, 2016), sendo o linfoma não-Hodgkin (LNH) a terceira neoplasia mais frequente em cães e a quinta em humanos dentre as neoplasias malignas (Zanini, 2013). Em ambas as espécies o LNH tem etiologia desconhecida e apresenta similaridade nos seus aspectos morfológicos, genotípicos e ainda na apresentação clínica e no prognóstico reservado, o que torna o linfoma canino (LC) um modelo translacional espontâneo para estudo do LNH em humanos (Zandvliet, 2016; Pittaway et al, 2019). Deste modo, os cães representam modelos excepcionais para definição de casualidade e compreensão de evolução, permitindo assim o desenvolvimento de novos protocolos terapêuticos para o linfoma, tais como os imunoterápicos (Dias et al, 2021).

Concomitantemente ao aumento da expectativa de vida dos animais domésticos, maiores vínculos afetivos entre os cães e seus responsáveis são observados, tais circunstâncias estreitaram muito os padrões de vida entre essas espécies, de modo que os cães efetivamente vivem em condições ambientais semelhantes às dos humanos (Giumelli \& Santos, 2016). Sendo assim, o LC pode ser particularmente adequado como um indicador de monitorização específico em situações ambientais de risco elevado para espécie humana, sendo considerada uma vantagem adicional, à adoção do animal como sentinela (Ito et al, 2014).

Os constantes estudos que avaliam a participação de fatores extrínsecos no surgimento das diversas desordens neoplásicas já permitiram elucidar uma série de fatores de riscos como: exposição aos raios ultravioletas, idade, condição reprodutiva, obesidade, entre outros (Bastos, 2014). Os fatores de risco consistem em algo que aumenta a probabilidade de um indivíduo desenvolver uma determinada doença ou sofrer um determinado agravo (Lauter et al, 2014).

Segundo Müller et al (2005) a prevalência global dos LNH varia de acordo com a região geográfica e situações temporais distintas. A exposição aos carcinógenos ambientais tem sido investigada e acredita-se que tenham forte participação na oncogênese do LC, entre eles destacam-se fumaça de tabaco (Pinello et al, 2017) e poluição do ar (Abba et al, 2011; Kliucininkas et al, 2011 \& Chakraborty, 2012).

Em decorrência da escassez de estudos aprofundados que retratem os aspectos epidemiológicos dos linfomas caninos na região nordeste do Brasil, avigora-se a necessidade da aplicação de ferramentas epidemiológicas para o avanço do conhecimento sobre os fatores que elevam a probabilidade de ocorrência desta neoplasia, bem como sua distribuição espacial. Deste modo, objetivou-se avaliar os aspectos epidemiológicos e a distribuição espacial dos casos de linfoma canino na Região Metropolitana do Recife-PE, Brasil.

\section{Metodologia}

\subsection{Aspectos Éticos}

O trabalho foi executado mediante autorização da Comissão de Ética no Uso de Animais (CEUA/UFRPE) sob Licença n. 65/2019. 


\subsection{Animais e Área de estudo}

O estudo consiste em uma pesquisa observacional do tipo caso-controle, realizada na Região Metropolitana do Recife, em que foram analisados dados epidemiológicos, obtidos de prontuários, referentes a 178 cães atendidos em clínicas veterinárias particulares localizadas na Região Metropolitana do Recife (RMR) -PE.

Adaptou-se o prontuário já utilizado pelas clínicas veterinárias, de modo que permitisse a coleta das informações clínicas e epidemiológicas de cada paciente, para execução do estudo. Profissionais atuantes nas clínicas veterinárias que compuseram o estudo foram capacitados no preenchimento dos prontuários clínicos, a fim de minimizar os erros.

Foram selecionadas duas clínicas, localizadas em cada um dos seguintes municípios: Recife, Olinda, Paulista, Cabo de Santo Agostinho e Jaboatão dos Guararapes. E uma clínica nos municípios de Araçoiaba, Camaragibe, Goiana, Ilha de Itamaracá, Igarassu, Ipojuca, Itapissuma, Moreno e São Lourenço da Mata, todos esses municípios componentes da Região metropolitana do Recife-PE.

Os animais do grupo caso (G1), grupo de cães com diagnóstico prévio de linfoma, foi constituído por 89 cães. Os critérios de inclusão no G1 foram: diagnóstico de linfoma obtido a partir do exame citopatológico, residir na RMR e ter o prontuário clínico devidamente preenchido. Ao passo que foram considerados como critérios de exclusão para composição do G1: animais que não residiam na RMR, pacientes que foram diagnosticados com outro(s) tipo(s) de neoplasia associada ao linfoma, cães com diagnóstico ou histórico clínico de outros tipos de neoplasia benigna e/ou maligna, bem como animais cujo prontuário encontrava-se incompleto.

O grupo controle (G2) foi composto por outros 89 cães. Para construção do G2 foram considerados os seguintes critérios de inclusão: prontuários de cães saudáveis atendidos no mesmo período dos cães do G1 e residir na RMR. Enquanto que os critérios de exclusão do G2 foram: Não residir na RMR, apresentar sinais clínicos de doenças infecciosas, metabólicas e/ou neoplásicas, cães com diagnóstico ou histórico clínico de neoplasia benigna e/ou maligna e pacientes com prontuários incompletos.

\subsection{Análise epidemiológica}

Para a análise dos dados dos animais do G1 empregou-se a dispersão das frequências absoluta e relativa nas seguintes variáveis: procedência do animal (com base no município e CEP informados), classificação citomorfológica do linfoma de acordo com Kiel (Lennert \& Feller, 1990) e Working Formulation (NCI, 1982), classificação anatômica, sítio primário e estadiamento clínico (Owen, 1980; Gray et al, 1984).

Para análise dos fatores de risco foram obtidas informações constantes nos prontuários, sobre idade, sexo, raça, possível contato deste com carcinógenos ambientais (solventes, inseticidas, tabaco), contato com humanos em tratamento quimioterápico/radioterápico, alimentação e moradia do animal. Para a variável raça os cães foram agrupados em: sem raça definida e com raça definida e a classificação por idade seguiu o padrão estabelecido por Fighera et al (2008), considerando: filhote (até um ano de idade), adulto (um a nove anos) e idoso (acima de 10 anos).

\subsection{Georreferenciamento}

Para a formulação do mapa da Região Metropolitana do Recife relacionado à distribuição dos casos de linfoma, os pontos de georreferenciamento foram obtidos a partir dos municípios, bairros e CEP's informados no prontuário clínico dos animais, com auxílio do programa MapCoordinates (MapCoordinates, 2019). Após a obtenção das coordenadas, estas foram lançadas no software Quantum GIS 2.8.2. para confecção do mapa. 


\subsection{Análise Estatística}

Para o estudo dos fatores de risco associados aos casos de LC diagnosticados foi realizada uma análise das variáveis de interesse pelo teste de Qui-quadrado de Pearson ou Exato de Fisher, quando necessário, e posteriormente, uma análise de regressão logística considerando como variável dependente os resultados do exame citopatológico. As variáveis independentes ou explanatórias consideradas no modelo foram aquelas que apresentaram significância estatística de $\mathrm{p}<0,05$. $\mathrm{O}$ programa EPIINFOTM 7.2.3. foi utilizado para a execução dos cálculos estatísticos.

\section{Resultados}

Os resultados das classificações citomorfológicas dos linfomas diagnosticados em cães estão expressos na Tabela 1.

Tabela 1 - Classificação citomorfológica dos linfomas caninos diagnosticados de acordo com as classificações de Kiel e Working Formulation.

\begin{tabular}{|c|c|c|c|}
\hline \multirow{2}{*}{ Kiel } & Diagnóstico & \multirow[t]{2}{*}{ Working Formulation } & Diagnóstico \\
\hline & Positivo (N/\%) & & Positivo (N/\%) \\
\hline \multicolumn{2}{|c|}{ Grau Baixo } & \multicolumn{2}{|c|}{ Grau Baixo } \\
\hline Linfocítico & $37 / 89(41,6 \%)$ & Linfocítico & $26 / 89(29,2 \%)$ \\
\hline Centrocítico & $20 / 89(22,5 \%)$ & \multicolumn{2}{|c|}{ Grau Intermediário } \\
\hline Centrocítico- centroblástico & $21 / 89(23,6 \%)$ & Células pequenas e clivadas & $27 / 89(30,3 \%)$ \\
\hline \multicolumn{2}{|c|}{ Grau Alto } & Misto de células pequenas e clivadas & $23 / 89(26 \%)$ \\
\hline Centroblástico & $7 / 89(7,9 \%)$ & Células grandes não clivadas & $8 / 89(9,0 \%)$ \\
\hline Imunoblástico & $3 / 89(3,4 \%)$ & \multicolumn{2}{|c|}{ Grau Alto } \\
\hline Linfoblástico & $1 / 89(1,0 \%)$ & Imunoblástico & $3 / 89(3,3 \%)$ \\
\hline \multirow[t]{2}{*}{ Anaplásico } & $0 / 89(0,0 \%)$ & Linfoblástico & $2 / 89(2,2 \%)$ \\
\hline & & Anaplásico & $0 / 89(0,0 \%)$ \\
\hline
\end{tabular}

Fonte: Autores.

A Tabela 1 retrata por meio de uma análise comparativa entre as classificações citomorfológicas de Kiel e Working Formulation, os diagnósticos citopatológicos de linfoma obtidos nos animais do estudo.

Quanto à classificação anatômica foi constatado que 58,4\% (52/89) dos casos de linfoma confirmados em cães foram do tipo multicêntrico, 19\% (17/89) classificados na forma cutânea, 10,1\% (9/89) na forma tímica, enquanto o tipo extranodal representou 8\% (7/89) do total de casos e o tipo alimentar 4,5\% (4/89).

Dentre os oitenta e nove cães diagnosticados com linfoma e incluídos na pesquisa, 58,4\% (52/89) teve como sítio primário do linfoma o próprio sistema linfático, em 19\% (17/89) a pele e/ou tecido subcutâneo foi o sítio primário da neoplasia seguidos pela região mediastinal com 10,1\% (9/89) do total de casos, olhos correspondendo a 3,3\% (3/89), órgãos do trato gastrointestinal em 4,5\% (4/89) dos casos, seguidos pelas fossas nasais e rins com 2,2\% (2/89) cada.

Em relação ao estadiamento clínico dos casos avaliados de linfoma canino, constatou-se que 5,6\% (5/89) foram estabelecidos como de Grau I (comprometimento de apenas um linfonodo); 12,4\% (11/89) de Grau II (comprometimento de vários linfonodos regionais); 44,9\% (40/89) de Grau III (linfoadenomegalia generalizada); 29,2\% (26/89) de Grau IV (comprometimento de fígado e/ou baço, com ou sem estágio I-III) e 7,9\% (07/89) de Grau V (comprometimento de medula óssea, com ou sem estágio I-III).

Com base nos resultados obtidos verificou-se que de acordo com a classificação por idade 70\% (62/89) dos casos de LC ocorreram em cães adultos, 28\% (25/89) em idosos e 2\% (02/89) em filhotes, com média de idade de oito anos. Cães com 
idade entre um a nove anos correspondem à faixa etária com maior risco de convalescer com $\mathrm{LC}(\mathrm{OR}=4,81 ; \mathrm{p}=0,000)$. Também foi constatado associação estatística ente LC e cães idosos, nessa faixa etária a probabilidade de ocorrência do LC é 3,40 vezes maior $(\mathrm{OR}=3,40 ; \mathrm{p}=0,010)$.

As fêmeas representaram 45\% (40/89) dos casos confirmados de LC, enquanto os machos 55\% (49/89). Não houve relação entre o sexo e o desenvolvimento do linfoma canino. Quanto à raça a população de cães com raça definida representou a maioria dos indivíduos avaliados, correspondendo a 63\% (56/89) do total, enquanto 37\% (33/89) dos casos ocorreram em cães sem raça definida. Ser caracterizado com de raça definida aumenta em 2,87 vezes as chances de desenvolvimento do linfoma ( $\mathrm{OR}=2,87 ; \mathrm{p}=0,000)$.

Quanto à procedência 58,4\% (52/89) dos animais eram provenientes do município de Recife (capital do estado), 19\% (15/89) do município de Olinda, 12,4\% (11/89) do município de Paulista, 3,3\% (3/89) de Jaboatão dos Guararapes e os municípios de Abreu e Lima, Ilha de Itamaracá e Camaragibe foram a procedência de 2,2\% (2/89) cada, dos animais analisados.

As demais variáveis independentes que apresentaram significância estatística de $p<0,05$ encontram-se expressas na Tabela 2.

Tabela 2 - Fatores de Risco associados aos linfomas caninos diagnosticados na Região metropolitana do Recife-PE.

\begin{tabular}{|c|c|c|c|c|c|}
\hline Variável & $\mathbf{N}$ & Diagnóstico Positivo (\%) & Valor de p & OR (IC 95\%) & Valor de $p$ \\
\hline \multicolumn{6}{|l|}{ Raça } \\
\hline SRD & 89 & $33(37,1 \%)$ & \multirow[b]{2}{*}{0,000} & \multirow{2}{*}{$2,87(1,57-2,29)$} & \multirow[b]{2}{*}{0,000} \\
\hline Com raça & 89 & $56(62,9 \%)$ & & & \\
\hline \multicolumn{6}{|l|}{ Sexo } \\
\hline Macho & 75 & $40(53,3 \%)$ & \multirow{3}{*}{0,271} & & \\
\hline Fêmea & 10 & $49(47,7 \%)$ & & & \\
\hline & 3 & & & & \\
\hline \multicolumn{6}{|l|}{ Idade (anos) } \\
\hline Filhote $(<1$ ano $)$ & 70 & $20(28,6 \%)$ & \multirow{3}{*}{0,000} & - & - \\
\hline Adulto (1 a 9 anos) & 82 & $54(65,9 \%)$ & & $4,81(2,41-9,61)$ & 0,000 \\
\hline Idoso (>9 anos) & 26 & $15(57,7 \%)$ & & $3,40(1,33-8,68)$ & 0,010 \\
\hline \multicolumn{6}{|l|}{ Habitação } \\
\hline Dentro de casa & 11 & $58(51,3 \%)$ & \multirow{3}{*}{0,377} & & \\
\hline & 3 & $58(51,3 \%)$ & & & \\
\hline Fora de casa & 65 & $31(47,7 \%)$ & & & \\
\hline \multicolumn{6}{|l|}{ Moradia } \\
\hline Apartamento & 82 & $35(425,7 \%)$ & \multirow{2}{*}{0,048} & \multirow{2}{*}{$1,72(0,95-3,13)$} & \multirow{2}{*}{0,072} \\
\hline Casa & 96 & $54(56,3 \%)$ & & & \\
\hline \multicolumn{6}{|c|}{ Habitação próxima à avenida ou rua movimentada } \\
\hline $\operatorname{Sim}$ & 79 & $56(70,9 \%)$ & \multirow[t]{2}{*}{0,000} & \multirow[t]{2}{*}{$4,86(2,56-9,23)$} & \multirow[t]{2}{*}{0,000} \\
\hline Não & 99 & $33(33,3 \%)$ & & & \\
\hline \multicolumn{6}{|l|}{ Água } \\
\hline Mineral & 82 & $35(42,7 \%)$ & \multirow{2}{*}{0,048} & \multirow{2}{*}{$1,29(0,68-2,45)$} & \multirow{2}{*}{0,420} \\
\hline Torneira & 96 & $54(56,3 \%)$ & & & \\
\hline \multicolumn{6}{|l|}{ Alimentação } \\
\hline Ração & 98 & $36(36,73 \%)$ & \multirow{3}{*}{0,001} & - & \multirow{3}{*}{0,014} \\
\hline Caseira & 46 & $27(58,7 \%)$ & & $2,44(1,19-5,00)$ & \\
\hline Mista & 34 & $26(76,5 \%)$ & & $5,59(2,29-13,66)$ & \\
\hline \multicolumn{6}{|l|}{ Contato com fumante } \\
\hline Sim & 45 & $23(51,1 \%)$ & \multirow[b]{2}{*}{0,500} & & \\
\hline Não & 13 & $66(49,6 \%)$ & & & \\
\hline Contato com humanos em & $\begin{array}{c}3 \\
\text { terap }\end{array}$ & e radioterapia & & & \\
\hline Sim & 14 & $13(92,9 \%)$ & & & \\
\hline Não & 16 & & 0,000 & $13,54(1,69-108,09)$ & 0,014 \\
\hline & 4 & $76(46,3 \%)$ & & & \\
\hline Contato com tintas/solvent & & & & & \\
\hline Sim & 19 & $16(84,2 \%)$ & & & \\
\hline Não & 15 & $73(45,9 \%)$ & 0,001 & $5,08(1,37-18,80)$ & 0,014 \\
\hline
\end{tabular}




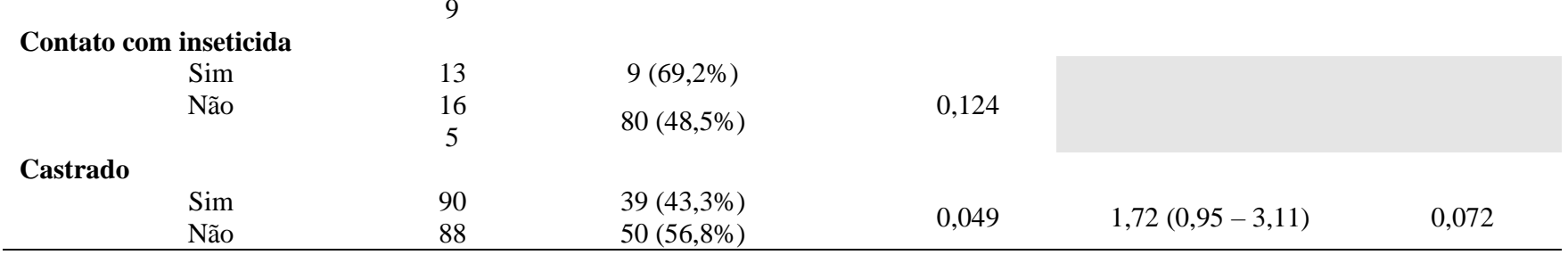

Fonte: Autores.

Na Tabela 2 são apresentadas as variáveis analisadas estatisticamente para estabelecimento dos fatores de riscos associados à ocorrência dos linfomas caninos, com cálculo da odds ratio (OR) que representa as chances de que um resultado ocorra em uma determinada exposição.

A distribuição espacial dos casos de linfoma canino na Região Metropolitana do Recife está representada na Figura 1.

Figura 1 - Distribuição espacial dos casos de linfoma canino na Região Metropolitana do Recife-PE.

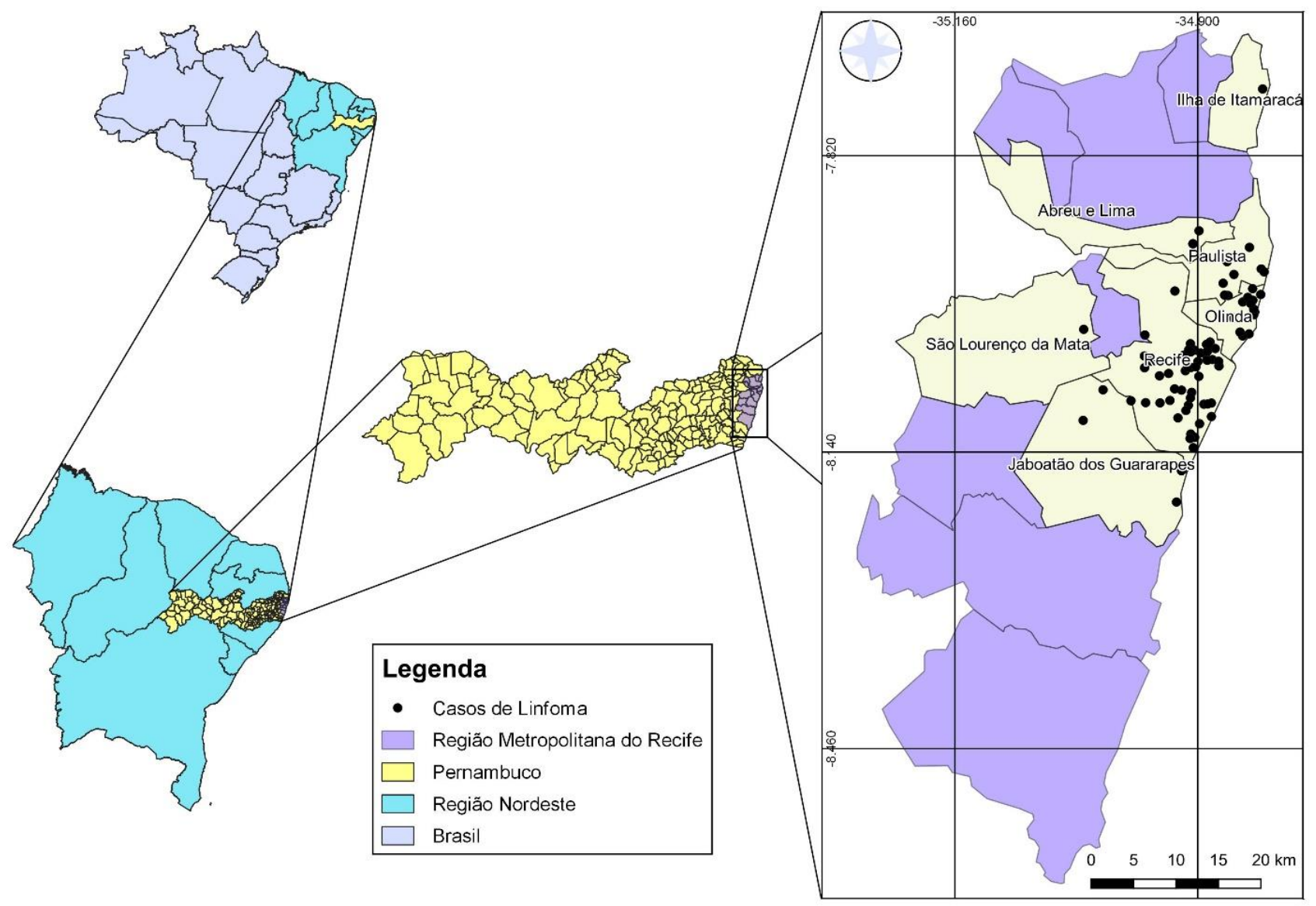

Fonte: Quantum GIS 2.8.2.

Na Figura 1 está representada em destaque a distribuição por municípios dos casos de linfoma canino diagnosticados na Região Metropolitana do Recife-PE 


\section{Discussão}

Nos cães, o linfoma é comumente classificado com base nos achados citológicos, sendo a análise citopatológica de grande valia no diagnóstico de linfoma em cães (Martini et al, 2022). Duas principais classificações são utilizadas para o LC: Kiel e Working Formulation, as mesmas utilizadas para LNH humano. A primeira indica o tempo de remissão da doença em pacientes tratados enquanto a segunda indica o tempo de sobrevida desses pacientes (Grandi et al, 2014).

Quando utilizada a classificação de Kiel predominaram os LC de grau baixo de malignidade (87,7\%), discordando do observado por Suzano et al (2010) que relatou predominância de LC com alto grau de malignidade. A classificação da Working Formulation quando aplicada revelou a predominância dos linfomas de grau intermediário $(63,6 \%)$, corroborando com Suzano et al (2010). Os resultados desse estudo também diferem dos encontrados por Teske e Van Heerder (1996), quando utilizaram as mesmas classificações em material colhido por PAAF. As características citomorfológicas dos linfomas nos pacientes avaliados permitiram incluí-las facilmente nas categorias descritas para as diferentes classificações de Kiel e Working Formulation, embora nem todos os tipos descritos em cada uma delas tenham sido diagnosticados revelando uma flutuação no tipo citomorfológico quando os resultados são comparados com os estudos acima descritos, essa flutuação pode estar relacionada à subjetividade nas análises, uma vez que a interpretação de qualquer exame pode sofrer com variações entre observadores distintos.

Quanto à classificação anatômica a maior prevalência de casos classificados como multicêntricos condiz com os relatos descritos anteriormente (Ponce et al, 2010; Kimura et al, 2011). Os linfomas multicêntricos são relatados como os mais prevalentes na espécie canina, acredita-se que por ter de maior evidência clínica facilita assim a observação pelos tutores e pelos próprios profissionais veterinários.

O estadiamento clínico, inicialmente elaborado para linfomas na espécie humana, norteia o prognóstico e tratamento dos linfomas na espécie canina, sendo realizado durante o diagnóstico clínico e seguindo o protocolo proposto pela Organização Mundial de Saúde (OMS). Os estádios variam de I a V de acordo com o grau de comprometimento de linfonodos e órgãos. A maior parte dos casos avaliados encontrava-se no estágio III (44,9\%), seguidos pelo estágio IV (29,2\%), diferentemente do observado por Dobson et al (2001) que relataram maior percentual em estádio clínico avançado (IV ou V) no momento do diagnóstico. A limitação dos tutores em identificar alterações clínicas preliminarmente minimiza o diagnóstico precoce do LC (Pittaway et al, 2019) ou ainda quando detectam, negligenciam o problema, reduzindo assim probabilidade de sucesso no tratamento e no tempo de sobrevida do animal.

O estudo dos fatores de risco consiste em importante modelo de probabilidade ou predição, de que um animal possa vir a desenvolver determinada afecção por um fator ou fatores específicos, propiciando com base nesta análise, intervenções diretas no que concerne a tomada de medidas preventivas. No caso dos linfomas em cães, há um compartilhamento desses fatores com os humanos, sendo esta espécie considerada sentinela (Gavazza et al, 2001). Neste estudo destacaram-se primordialmente fatores ambientais (contato com carcinógenos externos) e alimentares, faixa etária e raça.

A ocorrência, a distribuição geográfica e até mesmo o comportamento de tipos específicos de neoplasias estão relacionados a múltiplos fatores, incluindo sexo, idade, raça, predisposição genética e exposição aos carcinógenos ambientais (Rooyen et al, 2018).

Segundo Pastor (2009), a incidência do linfoma canino é progressiva, com aproximadamente 22 a 33 novos casos anuais para cada 100.000 cães e assim como nos casos de neoplasia em humanos, a progressão dos casos de linfomas em cães, tem sido constantemente associada aos fatores extrínsecos (Zanini, 2013). A maior incidência em cães adultos observada nesse estudo corrobora com o observado por Pittaway et al (2019), que ao avaliarem a incidência e os fatores de riscos associados ao linfoma canino no Reino Unido observaram a idade média global de 9 anos, semelhante ao observado neste estudo, em que cães categorizados como adultos ( 1 a 9 anos) apresentaram maior risco de desenvolverem LC (OR=4,81; $p=0,000)$, seguidos 
pelos cães senis (>9 anos) ( $\mathrm{OR}=3,40 ; \mathrm{p}=0,010)$. De modo geral, as neoplasias são desordens orgânicas da senilidade associadas ao desgaste metabólico e maior tempo de exposição a carcinógenos ambientais, portanto o avançar da idade tornase agravante para ocorrência dos linfomas.

Na década de 70, Dorn et al (1970) determinaram que não havia associação entre o sexo e a predisposição ao linfoma. No entanto, posteriormente foi estabelecida correlação positiva entre o status hormonal presuntivo e desenvolvimento de linfoma, sendo isto detectado tanto em cães quanto em humanos, visto que a ocorrência de linfoma foi menor em fêmeas não castradas e maior em mulheres que atingiram a menopausa (Villamil et al, 2009). Apesar de historicamente a ocorrência de linfoma canino ser considerada mais prevalente em machos do que em fêmeas, o sexo e o status de castração não são tradicionalmente associados ao aumento do risco de diagnóstico de linfoma (Pittaway et al, 2019).

Observou-se uma maior frequência de LC, neste estudo, em cães com raça definida. A menor variação genética em exemplares de raças definidas é a principal explicação para essa evidência, e essa realidade é observada em diversos locais do mundo ao passo que as associações entre raça e a ocorrência de linfoma canino, subsidiam pesquisas objetivando encontrar o modelo de raça canina mais adequado para realização de estudos comparativos com o LNH humano (Ruple-Czernieak, 2012).

Os riscos associados à raça para o desenvolvimento de linfoma canino diferem entre os países (Comazzi et al, 2018), reforçando a interferência ambiental na oncogênese deste e também evidenciando a importância de estudos epidemiológicos e de coleta de casos com dados geograficamente consistentes. Não se fez neste estudo, a avaliação estatística considerando as características de cada raça, pois o número de exemplares por raça não seria representativo. De acordo com a literatura algumas raças apresentam maior predisposição ao aparecimento de linfoma, tais como: bulldog, doberman, rottweiler, golden retrievers, bull terrier, terrier escocês e terrier branco das montanhas ocidentais (Yau et al, 2017; Pittaway et al, 2019).

O aumento do risco no surgimento de LC associado ao convívio de cães com pessoas em tratamento oncológico (quimioterapia e/ou radioterapia) pode estar relacionado a um conjunto de eventos decorrentes da terapia propriamente dita. De acordo com Instituto Nacional do Câncer do Ministério da Saúde (INCA, 2021), os quimioterápicos e radioterápicos são eliminados também pelas lágrimas e suor, sendo esses fluidos a principal forma de contato dos cães com os componentes carcinogênicos presentes nos recursos de terapia oncológica.

Outra hipótese dar-se, ao considerar as bruscas mudanças no convívio do paciente oncológico humano com seus cães. A Síndrome de Ansiedade por Separação (SAS) é amplamente discutida no âmbito do bem estar animal para espécie canina, por ser um subconjunto de problemas, decorrentes do estado de angústia. Os distúrbios comportamentais de cães associados à separação ocorrem na ausência do tutor de forma parcial ou absoluta (Bezerra \& Zimmermann, 2015). Fatidicamente a SAS culmina em estresse e imunossupressão, tornando o animal em estado de susceptibilidade ao linfoma ou outras enfermidades.

Segundo Bastos (2014), uma gama de agentes carcinogênicos químicos existe no meio ambiente e estão relacionados a hábitos sociais, alimentares e também ocupacionais. Os carcinógenos químicos como os derivados do tabaco e componentes de tintas e solventes como: azocorantes, aflatoxinas e benzeno são nitidamente associados ao surgimento de câncer no homem e animais (Gavazza et al, 2001; Tybjerg et al, 2018; Gatto, 2021). Gavazza et al (2001) observou maior incidência de LC em animais expostos continuamente à produtos químicos como tintas e solventes, corroborando com os achados encontrados neste estudo, em que o contato com tais produtos aumentou em cinco vezes as chances do surgimento do LC ( $\mathrm{OR}=5,08 \mathrm{p}=0,014)$. Sendo, portanto, um fator considerado de alto potencial à oncogênese de linfomas em cães.

Outro fator importante consiste na alimentação, a industrialização dos alimentos para animais é constantemente associada tanto ao aumento da expectativa dos cães quanto a influência na oncogênese de várias desordens neoplásicas, visto que diversos aditivos, ingredientes e contaminantes encontrados nas rações são carcinógenos potenciais. Entre eles destacamse pesticidas, metais pesados, o uso de resíduos de abatedouros, conservantes e corantes proibidos para consumo humano (Vasconcelos et al, 2013). Os questionamentos sobre a segurança das dietas comerciais aumentam ao passo que a medicina 
humana esclarece a influência de vários fatores alimentares com potencial carcinogênico, dentre eles destacam-se: obesidade, consumo de alimentos ultraprocessados com conservantes, glúten e açúcares refinados (Olgivie, 2006).

As frequentes dúvidas quanto à influência alimentar no desenvolvimento do LC assim como o de outras neoplasias tem contribuído para uma reconfiguração na proposta nutricional dos animais, e cada vez mais profissionais dedicam-se na elaboração de dietas que contemplem elementos nutricionais que cientificamente colaborem de forma preventiva e terapêutica, como vitamina E, ômegas e selênio que são potenciais antioxidantes (Case \& Daristotle, 2011). Segundo Vasconcelos et al (2013), se informações genéticas específicas relacionadas ao aparecimento de neoplasias fossem descobertas, seria possível a manipulação de dietas que auxiliassem na modulação das expressões gênicas, prevenindo o aparecimento da doença.

$\mathrm{O}$ risco aumentado no surgimento de LC associado ao consumo de alimentos naturais $(\mathrm{OR}=2,44)$ e de alimentação mista (ração+natural) $(\mathrm{OR}=5,59)$ neste estudo retrata que dicotomizar alimentação industrial como cancerígena e alimentação caseira como não cancerígena é um erro. Isso por que muitos tutores não seguem dietas naturais balanceadas por veterinários nutrólogos, ou seja, os animais avaliados estão expostos as mesmas condições alimentares associadas a neoplasias que os humanos. Em cães dietas desbalanceadas, comumente com altas concentrações de carboidratos, geram um estado de incompetência imunológica, além de ser fonte energética para que células neoplásicas iniciais evoluam para a doença clínica (Lonsdale, 2007).

O maior risco de cães, que residem próximos a ruas ou avenidas com alto fluxo automobilístico na RMR de desenvolverem linfoma $(\mathrm{OR}=4,86 ; \mathrm{p}=0,000)$, provavelmente deve-se a elevada quantidade de dióxido de carbono $(\mathrm{CO} 2) \mathrm{e}$ óxidos de nitrogênio produzidos por motores de veículos automotivos, que resultam do consumo de combustíveis, principalmente derivados do petróleo (Bastos, 2014). Esses compostos são sabidamente tóxicos ao ambiente, a saúde humana e animal (Papini, 2009; Drumm et al., 2014) com efeitos carcinogênicos conhecidos. Este fator de risco detectado, corrobora com os resultados de Zanini et al. (2013) em São Paulo, que também encontraram como fator de risco para o linfoma canino, o fato dos animais em suas habitações serem mantido ao ar livre e a 100 metros de ruas ou avenidas movimentadas (mais de 50 veículos por minuto) $(\mathrm{OR}=3,1 ; \mathrm{p}=0,005)$.

Ao observar o aumento do risco no desenvolvimento do linfoma em cães mediante a exposição aos fatores ambientais avaliados e considerando que tais animais estão inseridos no convívio humano, sugere-se que os mesmos fatores influenciem diretamente a saúde humana, reforçando a aplicabilidade de cães como principal sentinela para o LNH em humanos. Em contrapartida, a escassez de estudos epidemiológicos que avaliam a associação entre carcinógenos ambientais com o surgimento de linfoma e/ou outras neoplasias em animais é um fator limitante na elaboração de planos de contingência que auxiliem efetivamente na redução dos riscos que estão submetidos cães e humanos, que compartilham o mesmo ambiente.

O georreferenciamento consiste em outra importante ferramenta que subsidia os estudos epidemiológicos. Este estudo é inédito no estado considerando o uso da ferramenta na oncologia veterinária. A partir do momento que o espaço geográfico é visto não apenas como um espaço abstrato, sinônimo de superfície ou área geométrica (Czeresnia \& Ribeiro, 2000), os recursos conquistados com o uso do Sistema de Posicionamento Global (GPS) permitiram o fortalecimento dos estudos epidemiológicos.

Partindo dessa premissa, quando se analisa a distribuição espacial dos casos de linfoma canino na RMR, constata-se a presença de casos positivos distribuídos predominantemente na Região Sul do município de Recife e Norte do município de Olinda. Segundo a Federação das Indústrias do Estado de Pernambuco (FIEPE, 2012), 52,7\% das indústrias estão localizadas na RMR, com predominância nos seguimentos de confecção de artigos de vestuário e acessórios seguidos por indústria de fabricação de produtos alimentícios, ainda de acordo com Bastos (2014) o índice de urbanização no Estado é maior no litoral, principalmente na RMR. Dentre os municípios estudados, Recife detém a maior quantidade de veículos por habitante, segundo 
o IBGE (2018), enquanto Olinda é a quarta cidade com maior número de veículos circulantes. Todos esses elementos colaboram de forma decisiva na distribuição dos casos de linfoma em caninos na Região estudada.

\section{Conclusão}

Constata-se que ocorrência de linfomas caninos diagnosticados na Região Metropolitana do Recife-PE, concentra-se nas regiões sul e norte dos municípios de Recife e Olinda respectivamente e está intimamente relacionada à exposição de carcinógenos ambientais presentes no ar e alimentos, bem como no contato com quimioterápicos, tintas e solventes existentes no ambiente de moradia dos animais. Deste modo, credita-se a adoção de hábitos saudáveis e consequentemente uma melhor qualidade de vida propiciada aos animais, à redução na ocorrência dos linfomas caninos.

\section{Agradecimentos}

À Coordenação de Aperfeiçoamento de Pessoal de Nível Superior (CAPES).

\section{Referências}

Abba, E. J. (2011). Fine aerosol and PAH carcinogenicity estimation in outdoor environment of Mumbai City, India. Internal Journal Environmental Health Research, 22, 134-49. https://doi.org/10.1080/09603123.2011.613112

Bastos, L. N. V. (2014). Indicadores de saúde ambiental e incidência do câncer infanto-juvenil em Pernambuco (Dissertação de Mestrado). https://www.arca.fiocruz.br/handle/icict/12163

Bezerra, E. L., \& Zimmermann, M. (2015). Distúrbios Comportamentais em Cães: Ansiedade por Separação. Revista Científica REVET de Medicina Veterinária do UNICEPLAC, 2(1). http://revista.faciplac.edu.br/index.php/Revet/article/view/115/63

Case, L. P., \& Daristotle, L. (2011). Canine of Feline Nutrition. A Resource for Companion Animal Professionals. Elsevier Editora.

Chakraborty, J. (2012). Cancer risk from exposure to hazardous air pollutants: spatial and social inequities in Tampa Bay, Florida. Internal Journal Environmental Health Research, 22, 165-183. https://doi.org/10.1080/09603123.2011.628643

Comazzi, S., Marelli, S., Cozzi, M., Rizzi, R., Finotello, R., Henriques, J., Pastor, J., Ponce, F., Rohrer-Bley, C., Rütgen B. C., \& Teske E. (2018). Breedassociated risks for developing canine lymphoma differ among countries: an European canine lymphoma network study. BMC Veterinary Research, 14, 232. https://dx.doi.org/10.1186/s12917-018-1557-2.

Czeresnia, D., \& Ribeiro, A. M. (2000). O conceito de espaço em epidemiologia: uma interpretação histórica e epistemológica. Cadernos de Saúde Pública, 16 (3), 595-617. https://doi.org/10.1590/S0102-311X2000000300002

Dias, J. N. R., André, A. S., Aguiar, S. I., Gil, S., Tavares, L., \& Aires-da-Silva, F. (2021). Immunotherapeutic Strategies for Canine Lymphoma: Changing the Odds Against Non-Hodgkin Lymphoma. Frontiers in Veterinary Science, 8:621758. https://doi.org/10.3389/fvets.2021.621758

Dobson, J. M., Blackwood, L. B., Mcinnes, E. F., Bostock, D.E., Nicholls, P., Hoather, T. M., \& Tom, B. D. M. (2001). Prognostic variables in canine multicentric lymphosarcoma. Journal of Small Animal Practice, 42, 377-384. https://doi.org/10.1111/j.1748-5827.2001.tb02485.x

Drumm, F. C., Gerhardt, A. E., Eloi, A., Fernandes, G. D., Chagas, P., Sucolotti, M. S., \& Kemerich, P. D. C. (2014). Poluição atmosférica proveniente da queima de combustíveis derivados do petróleo em veículos automotores. Revista Eletrônica em Gestão, Educação e Tecnologia Digital, 18(1), 66-78. http://dx.doi.org/10.5902/2236117010537

FIEPE - Federação das Indústrias do Estado de Pernambuco (2012). FIEPE lança cadastro industrial de Pernambuco. https://www.leiaja.com/carreiras/2012/11/30/fiepe-lanca-cadastro-industrial-de-pernambuco/

Fighera, R. A., Souza, T. M., Silva, M. C., Brum, J. S., Graça, D. L., Kommers, G. D., Irigoyen, L. F., \& Barros, C.S.L. (2008). Causas de morte e razões para eutanásia de cães da Mesorregião do Centro Ocidental Rio-Grandense (1965-2004). Pesquisa Veterinária Brasileira, 28 (4), 223-230. https://doi.org/10.1590/S0100-736X2008000400005

Gatto, N. M. (2021). Environmental Carcinogens and Cancer Risk. Cancers, 13, 622. https://doi.org/10.3390/cancers1304062

Gavazza, A., Presciuttini, S., Barale, R., Lubas, G., \& Gugliucci, B. (2001). Association between canine malignant lymphoma, living in industrial areas, and use of chemicals by dog owners. Journal of Veterinary Internal Medicine, 15(3), 190-195. https://doi.org/10.1111/j.1939-1676.2001.tb02310.x

Giumelli, R. D., \& Santos, M. C. P. (2016). Convivência com animais de estimação: um estudo fenomenológico. Revista da Abordagem Gestáltica, 22(1), 4958. http://pepsic.bvsalud.org/scielo.php?script=sci_arttext\&pid=S1809-68672016000100007\&lng=pt\&tlng=pt

Gouveia, N., \& Prado, R. (2010). Spatial analysis of the health risks associated with solid waste incineration: a preliminary analysis. Revista Brasileira de Epidemiologia, 13(1), 3-10. https://doi.org/10.1590/S1415-790X2010000100001 
Grandi, F., Beserra, L. E. O., \& Costa, L. D. (2014). Citopatologia veterinária diagnóstica. MedVet Editora.

Gray, K. N., Raulston, G. L., Gleiser, C. A., \& Jardine, J. H. (1984). Histologic classification as an indication of therapeutic response in malignant lymphoma of dogs. Journal of American Veterinary Medical Association, 184, 814-817. https://europepmc.org/article/med/6725115

INCA - Instituto Nacional de Câncer José Alencar Gomes da Silva (2021). Quimioterapia. https://www.inca.gov.br/tratamento/quimioterapia

Ito, D., Frantz, A. M., \& Modiano, J. F. (2014). Canine lymphoma as a comparative model for human non-Hodgkin lymphoma: recent progress and applications. Veterinary immunology and immunopathology, 159(3-4), 192-201. https://doi.org/10.1016/j.vetimm.2014.02.016

Kimura, K. C., Zanini, D. A., Nishiya, A. T., Dias, R. A., \& Dagli, M. L. Z. (2011). Morphology and immunophenotypes of canine lymphomas: a survey from the service of animal pathology School of Veterinary Medicine and Animal Science, University of São Paulo, Brazil. Brazilian Journal of Veterinary Pathology, 4(3), 199-206. https://bjvp.org.br/wp-content/uploads/2015/09/DOWNLOAD-FULL-ARTICLE-34-20881_2011_11_13_35_59t-al.pdf

Kliucininkas, L. (2011). Indoor and outdoor concentrations of fine particles, particle-bound PAHs and volatile organic compounds in Kaunas, Lithuania. Journal of Environmental Monitoring, 1, p. 182-91, 2011. https://doi.org/10.1039/C0EM00260G

Lonsdale, T. (2007). Junk pet food and the damage done. Nexus Magazine, 14(6), 31-35.

Martini, V., Marano, G., Aresu, L., Bonfanti, U., Boracchi, P., Caniatti, M., Cian, F., Gambini, M., Marconato, L., Masserdotti, C., Nicoletti, A., Riondato, F., Roccabianca, P., Stefanello, D., Teske, E., \& Comazzi, S. (2022). Performance of lymph node cytopathology in diagnosis and characterization of lymphoma in dogs. Journal of veterinary internal medicine, 36(1), 204-214. https://doi.org/10.1111/jvim.16326

Miranda, R. C. (2007). Biodegradation of Diesel Oil by Yeasts Isolated from the Vicinity of Suape Port in the State of Pernambuco -Brazil. Brazilian Archives of Biology and Technology, 50, 147-152. https://doi.org/10.1590/S1516-89132007000100018

Müller, A. M., Ihorst, G., Mertelsmann, R., \& Engelhardt, M. (2005). Epidemiology of non-Hodgkin's lymphoma (NHL): trends, geographic distribution, and etiology. Annals of Hematology, 84, 1-12. https://doi.org/10.1007/s00277-004-0939-7

Olgivie, G.K. (2006). Nutrition and cancer: frontiers for cure. https://www.vin.com/apputil/content/defaultadv1. aspx?id=3859082\&pid=11223\&

Owen, L. N. (1980). TNM Classification of Tumours in Domestic Animals. https://apps.who.int/iris/handle/10665/68618

Papini, S. (2009). Vigilância em saúde ambiental: uma nova área da ecologia. Atheneu Editora.

Pastor, M., Chalvet-Monfray, K., Marchal, T., Keck, G., Magnol, J. P., Fournel - Fleury, C., \& Ponce, F. (2009). Genetic and Environmental Risk Indicators in Canine Non Hodgkin's Lymphomas: Breed Associations and Geographic Distribution of 608 Cases Diagnosed throughout France over 1 Year. Journal Veterinary Internal Medicine, 23(2), 301-310. https://doi.org/10.1111/j.1939-1676.2008.0255.x

Pinello, K. C., Santos, M., Leite-Martins, L., Niza-Ribeiro, J., \& de Matos, A. J. (2017). Immunocytochemical study of canine lymphomas and its correlation with exposure to tobacco smoke. Veterinary world, 10(11), 1307-1313. https://doi.org/10.14202/vetworld.2017.1307-1313

Pittaway, C., Schofield, I., Dobson, J., O’Neill, D. G., \& Brodbelt, D. C. (2019). Incidence and risk factors for the diagnosis of lymphoma in dogs in UK primary-care practice. Journal of Small Animal Practice, 60(10), 581-588. https://doi.org/10.1111/jsap.13054

Ponce, F., Marchal, T., Magnol, J. P., Turinelli, V., Ledieu, D., Bonnefont, C., Pastor, M., Delignette, M. L., \& Fournel-Fleury, C. (2010). A Morphological Study of 608 Cases of Canine Malignant Lymphoma in France With a Focus on Comparative Similarities Between Canine and Human Lymphoma Morphology. Veterinary Pathology, 47(3), 414-433. https://doi.org/10.1177/0300985810363902

Suzano, S. M. C., Sequeira, J. L., Rocha, N. S., \& Pessoa, A. W. P. (2010). Classificação citológica dos linfomas caninos. Brazilian Journal of Veterinary Research and Animal Science, 47(1), 47-54. http://www.revistas.usp.br/bjvras/article/view/26848

Teske, E., \& Van Heerde, P. (1996). Diagnostic value and reproducibility of fine needle aspiration cytology in canine malignant lynfoma. Veterinary Quaternaly, 18 (3), 112-115. https://doi.org/10.1080/01652176.1996.9694630

Tybjerg, A. J., Friis, S., Brown, K., Nilbert, M. C., Morch, L., \& Koster, B. (2018). Updated fraction of cancer attributable to lifestyle and environmental factors in Denmark in 2018. Scientific Reports, 12, 549 (2022). https://doi.org/10.1038/s41598-021-04564-2

Vasconcelos, A., Horta. R. S., \& Lavalle, G. E. (2013). Nutrição do paciente oncológico - uma visão integrativa. Cadernos Técnicos de Veterinária e Zootecnia, 70, 91-99. https://vet.ufmg.br/ARQUIVOS/FCK/file/editora/caderno\%20tecnico\%2070\%20oncologia\%20pequenos\%20animais.pdf

Villamil, J. A., Henry, C. J., Hahn, A. W., Bryan, J. N., Tyler, J. W., \& Caldwell, C.W. (2009). Hormonal and Sex Impact on the Epidemiology of Canine Lymphoma. Journal of Cancer Epidemiology, 2009, Article ID 591753, 7p. https://www.ncbi.nlm.nih.gov/pmc/articles/PMC2859020/pdf/JCE2009591753.pdf

Yau, P. P., Dhand, N. K., \& Thomson, P. C. (2017). Retrospective study on the occurrence of canine lymphoma and associated breed risks in a population of dogs in NSW (2001-2009). Australian Veterinary Journal, 95, 149-155. https://doi.org/10.1111/avj.12576

Zandvliet, M. (2016). Canine lymphoma: a review. Veterinary Quaternaly, 36(2), 76-104. https://doi.org/10.1080/01652176.2016.1152633.

Zanini, D. A., Kimura, K. C., Nishiya, A. T., Ubukata, R., Leandro, R. M., Brito, C. P., Trombetti, M., Lagoa, A. C., Macedo, T. R., Rodrigues, L. C. S., Rosendo, J. A. S., Arndt, H. L., Dias, R. A. D., \& Dagli, M. L. Z. (2013). Environmental risk factors related to the development of canine non-Hodgkin's lymphoma. Ciência Rural, 43(7), 1302-1308. https://doi.org/10.1590/S0103-84782013005000089 